\title{
Comparison of the haptic and visual deviations in a parallelity task
}

\author{
Astrid M. L. Kappers · Wouter B. Schakel
}

Received: 11 August 2010/Accepted: 16 November 2010/Published online: 5 December 2010

(C) The Author(s) 2010. This article is published with open access at Springerlink.com

\begin{abstract}
Deviations in both haptic and visual spatial experiments are thought to be caused by a biasing influence of an egocentric reference frame. The strength of this influence is strongly participant-dependent. By using a parallelity test, it is studied whether this strength is modality-independent. In both haptic and visual conditions, large, systematic and participant-dependent deviations were found. However, although the correlation between the haptic and visual deviations was significant, the explained variance due to a common factor was only $20 \%$. Therefore, the degree to which a participant is "egocentric" depends on modality and possibly even more generally, on experimental condition.
\end{abstract}

Keywords Reference frames - Haptic perception - Visual perception $\cdot$ Spatial perception $\cdot$ Parallel

\section{Introduction}

In daily life, it seems a necessary requirement to have an accurate spatial representation of the environment in order to interact with the objects surrounding us. However, perceptual spaces are often quite different from the corresponding physical spaces. As a consequence, perceptual judgements on spatial relations may be far from veridical. The distortion of visual space was already observed by Helmholtz (1867); Hillebrand (1902) and Blumenfeld (1913), although more recent studies have examined this phenomenon in much more detail (e.g., Cuijpers et al.

A. M. L. Kappers $(\bowtie) \cdot$ W. B. Schakel

Helmholtz Institute, Physics of Man, Utrecht University,

Padualaan 8, $3584 \mathrm{CH}$ Utrecht, The Netherlands

e-mail: a.m.1.kappers@uu.nl
2000; 2001; Doumen et al. 2005; Koenderink et al. 2000). Blumenfeld (1937) was also one of the first to observe the systematic deviations in the haptic domain and also von Skramlik (1937) reported on the distortion of haptic space. Recent studies have confirmed the nonveridicality of haptic space (e.g., Kappers 1999; 2003; Kappers and Koenderink 1999; Kaas and van Mier 2006; Newport et al. 2002).

The task most often used in the recent studies is to make two bars parallel, either visually with a remote control (e.g., Cuijpers et al. 2000, 2001; Doumen et al. 2005) or haptically while blindfolded (e.g., Kappers 1999, 2003; Kaas and van Mier 2006). The results have in common that the deviations are large, systematic and participantdependent. In the haptic case, when participants are asked to orient a test bar touched with their left hand in such a way that it feels parallel to a reference bar touched with their right hand and positioned $1.2 \mathrm{~m}$ away from the test bar, systematic deviations of more than $90^{\circ}$ were found for some individuals (e.g., Kappers 2003). Somewhat smaller (up to $40^{\circ}$ ) but still systematic deviations were reported in a visual parallelity task in a setting where the bars appeared at eye height at various distances from the observer and with large separation angles (Cuijpers et al. 2000).

There exists accumulating evidence, both psychophysical and neurophysiological, that the haptic deviations originate from the biasing influence of egocentric reference frames, which can either be hand-centered or bodycentered (e.g. Kappers 2003, 2004, 2007; Zuidhoek et al. 2003). For example, the definition of "parallel" in a handcentered reference frame would be that a bar always has the same orientation with respect to the hand, irrespective the location and orientation of that hand. If the participant's hand is placed in a natural way on the right side of a table, the orientation will be quite distinct from the orientation of the same hand placed at the left side. Consequently, bars 
parallel in this hand-centered reference frame would be far from parallel in the table reference frame (as is always the task in these experiments). The deviations found in the parallelity experiments are not as extreme as would be predicted by a complete reliance on an egocentric reference frame, but they are certainly biased in that direction.

The deviations found in the visual parallelity experiments might be understood in a similar way. In this case, the egocentric reference frame might be body-centered, eye-centered or head-centered. For example, think of looking at a bar aligned with the line of sight. In this case, we would only see one tip of the bar. However, if this bar was placed somewhere else, parallel to the original position, we would see a much larger part of this bar. If on the other hand, the bar would be made parallel in an egocentric reference frame, the bar has to be rotated such that also in this case only the tip is visible. Like in the haptic case, the deviations actually found are smaller than might be expected by performance in an egocentric reference frame, but they are biased in the predicted direction.

Thus, although participants in both the visual and haptic conditions are asked to make the bars parallel in an external reference frame (henceforth called allocentric reference frame), they are not sufficiently able to ignore their own egocentric reference frames. As a consequence, the parallelity settings lie in between veridical settings and those predicted by performance in an egocentric reference frame. The participant-dependent size of the deviations is determined by the degree to which the egocentric and the allocentric reference frames each contribute. Other studies support this possibility of a biasing influence of an egocentric reference frame (e.g., Paillard 1991; Soechting and Flanders 1992; Carrozzo and Lacquaniti 1994; Flanders and Soechting 1995; Gentaz et al. 2008).

The aims of the current study are twofold. First, as the participant-dependent deviations in both the haptic and visual studies are thought to be caused by egocentric biases, it is of interest to investigate whether these biases and thus these reference frames are related. If the degree to which a participant is more or less "egocentric" is modality-independent, participants with large (small) haptic deviations will also have large (small) visual deviations. This will be investigated using the same set-up in both conditions, so that the deviations can be compared directly. This set-up is similar to the one often used in the haptic studies, but is new for the visual condition. Therefore, the second aim of this study is to investigate visual spatial relations at short (i.e. reachable) distances. The hypothesis, based on informal observations in the laboratory, is that also under these conditions, systematic deviations will be found. As previous studies (e.g, Kappers 2003; Zuidhoek et al. 2007) found a significant gender difference in the size of the haptic deviations, equal numbers of women and men will participate.

\section{Method}

\section{Participants}

Participants were 16 men and 16 women, aged between 17 and 33 years (mean $22.3 \pm 3.2$ year). Most of them were students at Utrecht University. Four of the men were lefthanded, all other participants were right-handed according to Coren's questionnaire (1993). None of them had any known hand deficits, and their vision was normal or corrected to normal. All participants were naive as to the purpose of the experiment and signed a declaration of informed consent. They participated on a voluntary basis, and some of them received a small financial compensation for their efforts.

\section{Set-up}

The set-up consisted of two identical $78 \mathrm{~cm}$ high stools with a black five-star base. Square metal plates $(27 \times 27 \mathrm{~cm})$ were placed on top of the stools. The plates were covered with a plastic layer on which a protractor was printed. Aluminum bars with a length of $20 \mathrm{~cm}$ and a diameter of $1.1 \mathrm{~cm}$ were placed on the protractors; a small pin, which fitted in a hole in the center of the protractor, ensured that the bars could be rotated freely without being displaced. Small magnets attached to the bottom side of the bars slightly increased the resistance against movements to avoid unintended rotations (hence the use of the iron plate). At one end, the bars ended in an arrow-shape so that the orientation could be read off easily with an accuracy of about $0.5^{\circ}$.

The distance between the two stools (and thus also between the centers of the two protractors) was $120 \mathrm{~cm}$. A third stool, on which the participant could be seated, was placed in the center and in front of the line between the two protractors. In this way, the position of the participant with respect to the two bars mimicked that of previous haptic studies (e.g., Kappers 2003). The third stool could be adjusted in height, such that shoulder height while seated was $110 \mathrm{~cm}$ for all participants.

The experimental room was painted black to reduce the visual information about the environment. In the visual condition, a thin circular sheet of black cardboard was placed between the plastic layers and the bars, so that the participant could not see the protractor or the plate. By lifting this sheet slightly, the experimenter was still able to read off the orientation of the bars. 
Stimuli

In both haptic and visual conditions, 8 reference orientations were used: $20^{\circ}, 40^{\circ}, 60^{\circ}, 80^{\circ}, 100^{\circ}, 120^{\circ}, 140^{\circ}$, and $160^{\circ}$. The $0^{\circ}$ orientation was defined as pointing to the right; increasing angular values signified a counterclockwise rotation. The main reason for using different reference orientations was to provide variation in the stimulus set. All reference orientations were presented four times to each participant in each condition, twice placed on the right protractor and twice on the left protractor.

\section{Procedure}

Before the experiment started, it was confirmed that the participant correctly understood the notion "parallel". They were asked to position two pens parallel with respect to each other on a table. They were urged to do so for an oblique orientation at a relatively large distance between the pens. All participants passed this test.

When participants entered the experimental room, the set-up was covered. They seated themselves on the stool, and its height was adjusted. Half of the participants ( 8 men and 8 women) started with the visual condition. Each condition consisted of two series of 16 trials on which the reference bar was placed on the right or the left protractor, respectively. The order of these series was counterbalanced over participants and gender. The order of the trials within a series was random. The start orientation of the test bar was chosen randomly within one of the four quadrants; within a series, all quadrants occurred equally often. Finishing both conditions took about $1 \mathrm{~h}$ per participant.

\section{Haptic condition}

Participants blindfolded themselves, after which the cover over the set-up was removed. By moving the wrist of the participant, the experimenter placed one hand of the participant above the reference bar and the other hand above the test bar. The right hand always touched the right bar and the left hand always the left bar. Participants were free to rotate their hands as they preferred; many of the participants tried to align their hands with that of the bars, but for many of the reference orientations, this was not possible. The participant was asked to rotate the test bar in such a way that it felt parallel to the reference bar. Maximum time per trial was about $20 \mathrm{~s}$, but in practice much less. When the participant indicated by lifting his/ her arms that $\mathrm{s} / \mathrm{he}$ was satisfied with the setting, the experimenter noted down the adjusted orientation and prepared the next trial.
Visual condition

In this condition, participants were not allowed to touch the bars. They had to instruct the experimenter how to rotate the test bar in such a way that the two bars looked parallel. The experimenter was standing somewhat behind the participant, so that the participant mainly saw the arm and hand of the experimenter. The participant could look back and forth between the test and the reference bars. When the participant was satisfied with the setting, s/he said so and then looked away. The experimenter could lift the cardboard that covered the protractor and noted down the adjusted orientation. Also in this condition, the maximum duration of a trial was about $20 \mathrm{~s}$.

Analysis

From previous studies (e.g., Kappers 2003), it is known that in the haptic condition, the deviations will mostly be clockwise if the test bar is on the right and counterclockwise if the test bar is on the left. If one ignores whether a bar is a test bar or a reference bar, this means that the right bar is always rotated clockwise with respect to the left bar. Therefore, it makes sense to define the deviations as the orientation of the left bar minus that of the right bar, irrespective whether the bar is a reference or a test bar. Following this definition, the expected deviations in the haptic case will mostly be positive and signed deviations can be used in the analyses. Based on informal observations, it seems legitimate to use the same definition in the visual condition.

When deviations in the visual and haptic conditions are compared, paired $T$ tests will be used. For the comparisons of men and women, unpaired $T$ tests will be used. This test will be two-sided in the visual case, as there are as yet no expectations for the influence of gender. The influence of reference orientation will be tested by means of a repeated measures Analysis of Variance (ANOVA). If sphericity is violated, Greenhouse-Geisser correction will be applied. Bonferroni correction will be used for subsequent multiple comparisons. Errors indicated in the text are standard errors.

\section{Results}

For each participant, the deviation averaged over all eight reference orientations and both the left and right reference locations is shown in Fig. 1 for both the visual and the haptic condition. Data of the participants are ordered according to the average haptic deviation of a participant. The average haptic deviation is $57.8^{\circ} \pm 2.5$ and ranges from $29.8^{\circ}$ to $79.0^{\circ}$. Men and women have deviations of 
Fig. 1 Deviations obtained in the haptic and visual conditions for all 32 participants, ordered by the size of the deviation in the haptic condition. Deviations are averaged over all 32 trials of a participant. Error bars indicate standard deviations

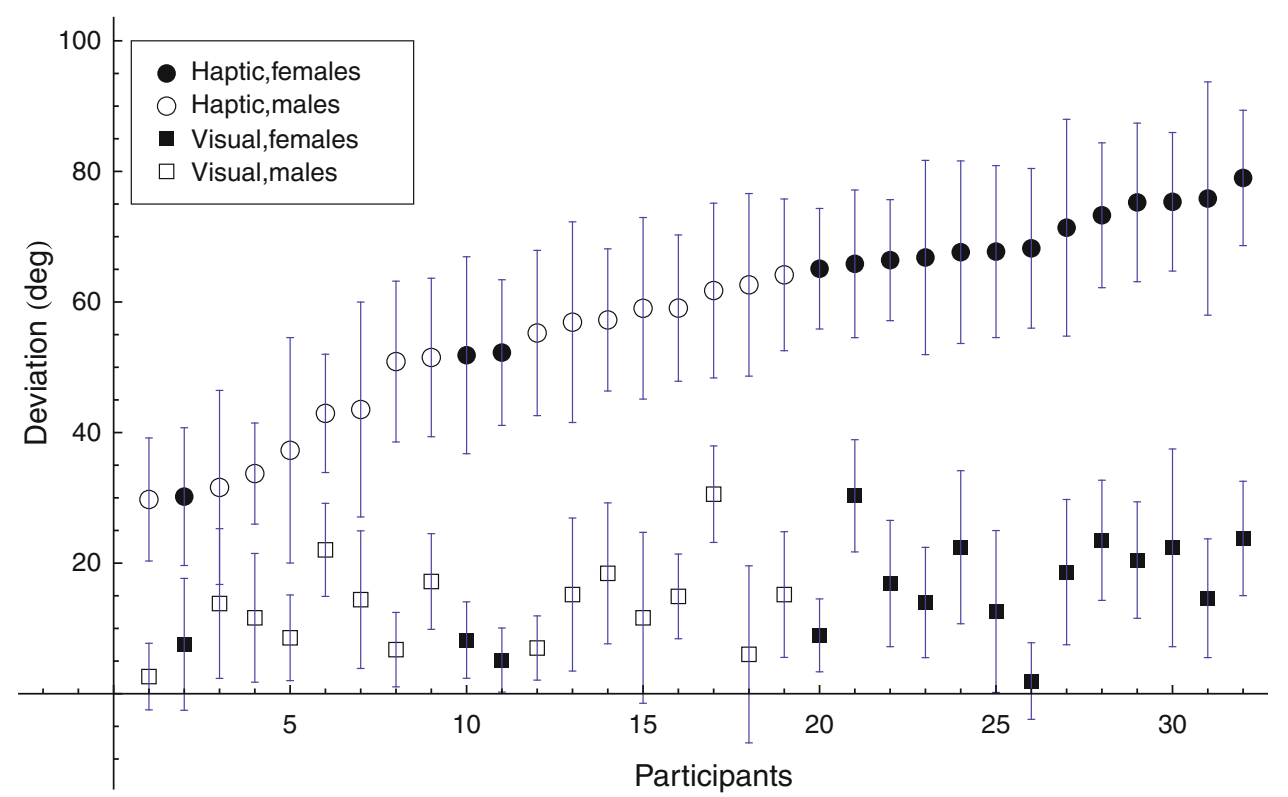

$49.8^{\circ} \pm 2.9$ and $65.8^{\circ} \pm 3.0$, respectively. This difference between men and women is highly significant $(P<0.0005)$. Interestingly, the visual deviations are also positive, and thus in the same direction, The average visual deviation is $14.6^{\circ} \pm 1.3$ and ranges from $1.9^{\circ}$ to $30.6^{\circ}$. Men and women have deviations of $13.5^{\circ} \pm 1.7$ and $15.7^{\circ} \pm 2.0$, respectively. This slight difference between men and women is far from significant $(P>0.8)$. From Fig. 1, it is already clear that the haptic deviations are much larger than the visual deviations, and this difference is indeed significant $(P<0.0001)$.

One of the research questions was to investigate whether the participant-dependent deviations found in the haptic and visual conditions are correlated. It turns out that a linear relationship between the visual and haptic deviations is significant $(P<0.05)$ with correlation $R=0.45$. This correlation is also significant for the female participants separately $(R=0.55, P<0.05)$, but not so for the men $(R=0.32, P>0.2)$.

In Fig. 2, the deviations as a function of the reference orientation are shown for both the haptic and visual conditions and for men and women separately. A Mixed ANOVA of the haptic deviations with reference orientation as within-subjects factor and gender as between-subjects factor revealed a significant effect of orientation $(F(142.44,4.75)=2.89, P=0.018)$. However, none of the pairwise comparisons reached significance. Not surprisingly, the main effect of gender was significant $(F(1,30)=14.29, P=0.001)$. Separate ANOVAs on the male and female deviations show that for the women, the effect of orientation is significant $(F(3.77,56.53)=$ $4.18, P=0.06$ ). Subsequent pairwise comparisons showed that only the difference between reference orientations $20^{\circ}$ and $60^{\circ}$ was significant. For the men, the effect of orientation was not significant $(P>0.8)$. The correlation between the male and female deviations per reference orientation is $R=0.54(P>0.15)$. There was no significant interaction between reference orientation and gender.

It can already be seen in Fig. 2 that the effect of reference orientation is significant in the visual condition. A Mixed ANOVA shows that the effect of orientation is indeed significant $(F(3.49,100.82)=65.22, P<0.001)$. Many of the subsequent pairwise comparisons reached significance, the major ones being that the deviations belonging to $80^{\circ}$ and $100^{\circ}$ were significantly different from all the others except each other, and $140^{\circ}$ was different from all the others except $40^{\circ}$. It can also be seen in Fig. 2 that the female deviations are higher than those of the males for each reference orientation, but this effect of gender was not significant $(P>0.4)$. The correlation between the male and female deviations per reference orientation was high: $R=0.98(P<0.0001)$. There was no significant interaction between reference orientation and gender.

\section{Discussion}

The deviations found in the haptic condition are in full agreement with those reported in earlier studies (e.g., Kappers 2003): the deviations are large (on average 57. $8^{\circ}$ ), systematic (the right bar is always rotated clockwise with respect to the left bar) and participant-dependent (average deviations range from $29.8^{\circ}$ to $79.0^{\circ}$ ). Moreover, female participants have significantly larger deviations than male participants, which also reproduces previous results (e.g, Kappers 2003; Zuidhoek et al. 2007). 
Fig. 2 Visual and haptic deviations as a function of reference orientation and averaged over all female or male participants. Error bars indicate standard errors

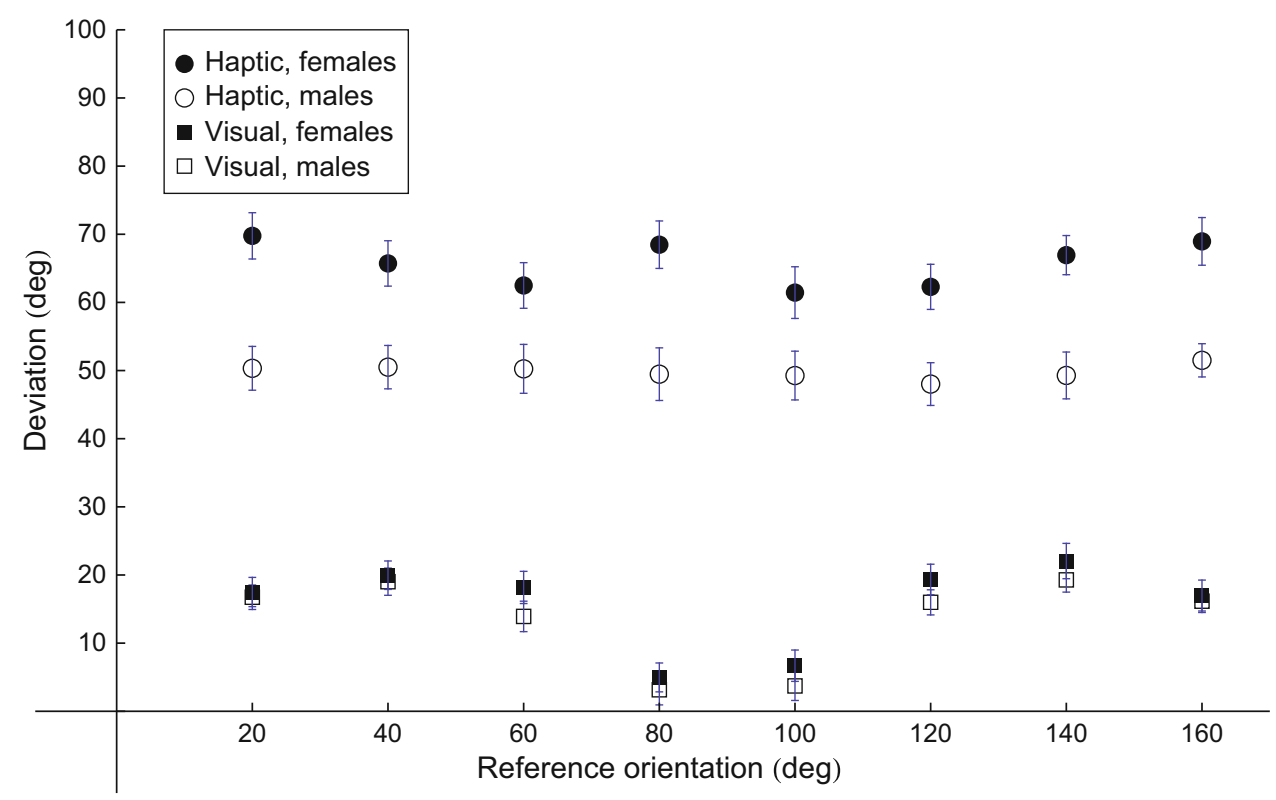

Of more interest are the results of the visual condition, as these have not been measured before. Although the deviations are smaller than those in the haptic condition, they follow a similar pattern: the deviations are relatively large (on average $14.6^{\circ}$ ), they are systematic in the same way as the haptic deviations, and they are participantdependent (average deviations range from $1.9^{\circ}$ to $30.6^{\circ}$ ). Although Fig. 2 may suggest otherwise, there are no significant differences between the deviations of female and male participants. The deviations are consistent with the hypothesis that perceptual decisions are made in a reference frame intermediate to an egocentric and an allocentric reference frame: body-centered, head-centered as well as eye-centered reference frames would bias the settings in the observed direction.

In both haptic and visual conditions, the influence of reference orientation was significant, albeit in quite different ways. In the haptic condition, this effect was not very pronounced as none of the pairwise comparisons reached significance. Previous haptic studies (e.g. Kappers 2003) showed clear effects of reference orientation if cardinal orientations $\left(0^{\circ}\right.$ and $\left.90^{\circ}\right)$ were compared to oblique orientations $\left(45^{\circ}\right.$ and $\left.135^{\circ}\right)$ : participants with a weak egocentric bias showed an oblique effect (i.e. smaller deviations for cardinal orientations than for oblique orientations), whereas participants with a strong egocentric bias showed the reversed effect. As these cardinal and oblique orientations were not used in the present study and since different orientations might have opposing effects on participants depending on the strength of their egocentric reference frame, no large orientation effects were to be expected. In the visual condition, the deviations for the $80^{\circ}$ and $100^{\circ}$ orientations are significantly smaller than all other deviations, which might be caused by the visibility of the walls to which these orientations are almost aligned. Cuijpers et al. (2001) showed that in their visual parallelity experiment, participants were influenced by external references such as the walls, even though they were covered with wrinkled plastic.

It is of interest to compare the current visual deviations obtained within graspable space to those obtained in more remote conditions. In the study by Cuijpers and colleagues (2000), the bars were presented at eye level at distances between 1.47 and $4.31 \mathrm{~m}$. Deviations did not depend on the distance to the observer, but there was a linear dependency on separation angle (ie, the visual angle between the two bars): the larger the separation angle, the larger the deviation. The deviations were strongly participant-dependent, both in the size of the deviations (up to $40^{\circ}$ ) as well as their dependence on reference orientation. Doumen and colleagues (2005) repeated this study under free viewing conditions in a somewhat richer context, and they found basically the same results. The deviations found in the current study seem to be smaller than those in previous studies, even though our separation angle was much larger. The most obvious cause is the difference in view point. When looking at bars at eye level, the length of the projection of the bar on the retina changes substantially with rotation of the bar. When looking at these bars from above, the effect of rotation of the bars on the projection on the retina is different, since not only the length changes but also the orientation. It is well possible that in this case, context information from the walls of the room helps to reduce the deviation.

The fact that the visual deviations were smaller than the haptic deviations might have been expected, as none of the 
previous visual studies in different experimental conditions reported deviations as large as sometimes found in the haptic studies. Moreover, in a very different experiment on the comparison of perceptual spaces, Lechelt et al. (1976) also found that visual errors were smaller than haptic errors. By varying the experimental conditions, it is possible to manipulate the balance between the egocentric and allocentric reference frames (e.g., Rossetti et al. 1996). In haptic experiments, for example, it has been shown that non-informative vision strengthens the influence of the allocentric reference frame, and therefore, it has a beneficial effect on haptic processing (Newport et al. 2002; Zuidhoek et al. 2004; Volcic et al. 2008). Non-informative vision was, of course, available in all above-mentioned visual experiments as well as in the present study, so this might be one of the causes of smaller deviations in comparison with the haptic deviations. It might even be questioned whether the visual information given by the walls should be termed non-informative in our study. When viewing the bars from above, their orientation might be compared (either consciously or unconsciously) to that of the walls.

One might wonder why there is a significant gender effect in the haptic condition and not in the visual condition. Many similar haptic studies also show this gender effect (e.g, Kappers 2003; Zuidhoek et al. 2007), but in the visual studies in this field, only few participants (typically 4) took part, and gender was often not even reported (e.g., Cuijpers et al. 2000, 2001; Doumen et al. 2005; Koenderink et al. 2000). Thus, although the deviations reported in these visual studies were often participant-dependent, it is unclear whether there was any influence of gender. We find this difference between the visual and the haptic conditions interesting and intruiging, but we find it too early to speculate about possible causes.

Although the weighting of the influence of the egocentric and allocentric reference frames may be manipulated by the experimental conditions, there is also a strong dependence on the participant: the deviations in both the visual and the haptic experiments range widely over the participants, and this is assumed to be caused by a different weighting of the reference frames. It was therefore a major aim of this study, to investigate whether this participantdependent weighting would be modality-independent. If that would be the case, the haptic and visual deviations measured in the same set-up should be correlated. However, although this correlation of $R=0.45$ was significant, the explained variance due to a common factor in the visual and haptic conditions is only $20 \%\left(R^{2}=0.2\right)$. Apparently, the degree to which a participant is more or less "egocentric" is not fixed, but depends on the modality and possibly even on the experimental condition.
Acknowledgments This research was supported by a grant from the Netherlands Organisation for Scientific Research (NWO).

Open Access This article is distributed under the terms of the Creative Commons Attribution Noncommercial License which permits any noncommercial use, distribution, and reproduction in any medium, provided the original author(s) and source are credited.

\section{References}

Blumenfeld W (1913) Untersuchungen über die scheinbare Grösse im Sehraume. Z Psychol 65:241-404

Blumenfeld W (1937) The relationship between the optical and haptic construction of space. Acta Psychol 2:125-174

Carrozzo M, Lacquaniti F (1994) A hybrid frame of reference for visuo-manual coordination. Neuroreport 5:453-456

Coren S (1993) The left-hander syndrome. Vintage Books, New York

Cuijpers RH, Kappers AML, Koenderink JJ (2000) Large systematic deviations in visual parallelism. Perception 29:1467-1482

Cuijpers RH, Kappers AML, Koenderink JJ (2001) On the role of external reference frames on visual judgements of parallelity. Acta Psychol 108:283-302

Doumen MJA, Kappers AML, Koenderink JJ (2005) Visual space under free viewing conditions. Percept Psychophys 67:1177-1189

Flanders M, Soechting JF (1995) Frames of reference for hand orientation. J Cognitive Neurosci 7:182-195

Helmholtz H von (1867/1962) Treatise on physiological optics volume 3 (New York: Dover, 1962); (English trans: Southall JPC) for the Optical Society of America (1925) from the 3rd German edition of Handbuch der physiologischen Optik (first published in 1867, Leipzig: Voss)

Gentaz E, Baud-Bovy G, Luyat M (2008) The haptic perception of spatial orientations. Exp Brain Res 187:331-348

Hillebrand F (1902) Theorie der scheinbaren Grösse bei binocularem Sehen. Denkschriften der Wiener Akademie. MathematischNaturwissenschaftliche Klasse 72:255-307

Kaas AL, van Mier HI (2006) Haptic spatial matching in near peripersonal space. Exp Brain Res 170:403-413

Kappers AML (1999) Large systematic deviations in the haptic perception of parallelity. Perception 28:1001-1012

Kappers AML (2003) Large systematic deviations in a bimanual parallelity task: further analysis of contributing factors. Acta Psychol 114:131-145

Kappers AML (2004) The contributions of egocentric and allocentric reference frames in haptic spatial tasks. Acta Psychol $117: 333-340$

Kappers AML (2007) Haptic space processing-allocentric and egocentric reference frames. Can J Exp Psychol 61:208-218

Kappers AML, Koenderink JJ (1999) Haptic perception of spatial relations. Perception 28:781-795

Koenderink JJ, van Doorn AJ, Lappin JS (2000) Direct measurement of curvature of visual space. Perception 29:69-79

Lechelt EC, Eliuk J, Tanne G (1976) Perceptual orientational asymmetries: a comparison of visual and haptic space. Percept Psychophys 20:463-469

Newport R, Rabb R, Jackson SR (2002) Noninformative vision improves haptic spatial perception. Curr Biol 12:1661-1664

Paillard J (1991) Motor and representational framing of space. In: Paillard J (eds) Brain and Space. Oxford University Press, Oxford, pp 163-182

Rossetti Y, Gaunet F, Thinus-Blanc C (1996) Early visual experience affects memorization and spatial representation of proprioceptive targets. Neuroreport 7:1219-1223 
Volcic R, van Rheede JJ, Postma A, Kappers AML (2008) Differential effects of non-informative vision and visual interference on haptic spatial processing. Exp Brain Res 190:31-41

von Skramlik E (1937) Psychophysiologie der Tastsinne. Akademische Verlagsgesellschaft, Leipzig

Soechting JF, Flanders M (1992) Moving in three-dimensional space: frames of reference, vectors, and coordinate systems. Annu Rev Neurosci 15:167-191

Zuidhoek S, Kappers AML, van der Lubbe RHJ, Postma A (2003) Delay improves performance on a haptic spatial matching task. Exp Brain Res 149:320-330
Zuidhoek S, Visser A, Bredero ME, Postma A (2004) Multisensory integration mechanisms in haptic space perception. Exp Brain Res 157:265-268

Zuidhoek S, Kappers AML, Postma A (2007) Haptic orientation perception: sex differences and lateralization of functions. Neuropsychologia 45:332-341 